\title{
Effect of gold nanoparticles on the expression of efflux pump mex $A$ and mex $B$ genes of Pseudomonas aeruginosa strains by Quantitative real-time PCR
}

\author{
Keramat Dorri ${ }^{1}$, Farzan Modaresi ${ }^{2}$, Mohammad Reza Shakibaie $^{3}$, Elham Moazamian $^{1}$ \\ 1 Department of Microbiology, College of Sciences, Agriculture and Modern Technology, Shiraz Branch, Islamic Azad University, Shiraz, Iran \\ 2 Department of Bacteriology and Virology, Jahrom Medical School, Jahrom University of Medical Sciences, Jahrom, Iran \\ 3 Department of Microbiology and Virology, Kerman University of Medical Sciences, Kerman, Iran \\ Corresponding author: Farzan Modaresi (modaresi7079@gmail.com)
}

Received 4 November 2021 • Accepted 7 December 2021 • Published 25 January 2022

Citation: Dorri K, Modaresi F, Shakibaie MR, Moazamian E (2022) Effect of gold nanoparticles on the expression of efflux pump mexA and mexB genes of Pseudomonas aeruginosa strains by Quantitative real-time PCR. Pharmacia 69(1): 125-133. https://doi. org/10.3897/pharmacia.69.e77608

\begin{abstract}
Antibiotic-resistant Pseudomonas aeruginosa infections are usually difficult to treat, and there are limited antibiotics for treating them. Increased antibiotic resistance of this bacterium, especially in a multidrug form, has caused many problems for treatment. Nowadays, metal nanoparticles are considered as appropriate alternatives to antibiotics. The objective of the present study was to investigate the effect of gold nanoparticles on the expression of MexB and MexA genes in Pseudomonas aeruginosa isolates.Pseudomonas aeruginosa isolate was identified using biochemical tests and an API kit. The antibiotic sensitivitytest for different antibiotics was performed withthe Kirby-Bauer test according to the CLSI standard. The presence of MexB and MexA genes was assessed by PCR. The effect of gold nanoparticles was investigated by microdilution to evaluate the minimum inhibitory concentration, and the expression of $M e x B$ and MexA treated genes was done with silver nanoparticles by the Real-Time PCR method.40 Pseudomonas aeruginosa isolates were detected and identified. These isolates showed significant resistance to various antibiotics. All strains were carriers of MexB and MexA genes, and finally, in the expression of MexB and MexA genes, a significant decrease in the expression of these genes was observed in the samples treated with gold nanoparticles compared to non-treated samples. One of the mechanisms of antibacterial activity of gold nanoparticles is through reducing the expression of mexA and mexB genes and thus reducing the number of active efflux pumps at the cell surface.
\end{abstract}

\section{Keywords}

Pseudomonas aeruginosa,gold nanoparticles, MexA, MexB, Real Time PCR

\section{Introduction}

Pseudomonas aeruginosa is a gram-negative opportunistic pathogen that is a major cause of death in immunocompromised patients due tothe creation of life-threatening infections (Sadikot et al. 2005; Gellatly et al. 2013). Pseudomonas aeruginosa is one of the main causes of septicemia, urinary tract infection, and endocarditis, as well as skin, eye, and ear infections in hospitalized burn-injured patients (Ghazi et al. 2012; Jafari et al. 2013). Pseudomonas aeruginosa is resistant to antimicrobials through various mechanisms; for instance, chromosomal mechanisms of antibiotic resistance, induced 
by efflux pump systems, have been reported in the membrane of this microorganism (Munita and Arias 2016). Pseudomonas aeruginosa has the potential to express 12 types of multidrug leakage pumps called Mex-Type Multidrug Resistance Efflux Pump; the five of them named MexAB-oprM, MexXYoprM, MexEF-oprN, MexCD-oprT, and MexJK-oprM are the most significant factors of resistance to antibiotics. MexAB-oprM is the most important system for removing antimicrobial compounds from the cell and resolving inherent drug resistance in Pseudomonas aeruginosa (Goli et al. 2018). Moreover, unlike other pumps that are of the acquired type and less important clinically, MexAB-oprM is the only leakage pump that is inherently expressed in wild strains (Nehme and Poole 2007). This situation prompts the development of alternative therapeutic strategies for bacteria, and if appropriate inhibitors are used, it can disrupt the function of these pumps to inhibit multidrug resistance. Due to their antimicrobial potential, some nanoparticles, such as gold nanoparticles, can be effective as an alternative method of antibiotics to fight bacterial infections. Gold has antibacterial, viral, and fungal properties (Kalishwaralal et al. 2010). Gold nanoparticles can also simultaneously affect vital parts of the microbial cell such as protein, energy, and DNA production(Rai et al. 2009). The purpose of the present study is to determine the antibacterial effect of gold nanoparticles on the expression of important genes of the MexAB-oprM efflux pump system called mexA and mexB in Pseudomonas aeruginosa isolates.

\section{Materials and methods}

The present study was conducted on Pseudomonas aerugino$s a$ strains in patients suspected of having Pseudomonas aeruginosa infections and referred to hospitals and health centers in the south of Fars province, Iran, within 12 months from September 2018 to September 2019. The clinical samples were burn, throat, nose, and 6 from the referred patients.

\section{Identification and detection}

Identification and detection of Pseudomonas were made biochemically using an API kit. In the biochemical method, gram staining and biochemical detection tests including oxidase, evaluation of fermentation on TSI, oxidation of glucose in the OF medium, evaluation of motility on SIM medium, indole, growth at $42{ }^{\circ} \mathrm{C}$, and arginine dihydrolase tests, were performed to identify and detect Pseudomonas aeruginosa. In the method of using API kit, Pseudomonas aeruginosa samples were confirmed, and Pseudomonas aeruginosa ATCC27853 was considered as a positive control.

\section{Antibiotic sensitivity}

In order to evaluate the antibiotic sensitivity of Pseudomonas aeruginosa strains, the disc diffusion method was used by employing Mueller-Hinton agar culture medium as a growth medium for bacteria and antibiotic discs Ciprofloxacin,Imipenem,Amikacin,Aztreonam, Cefepime, Ceftazidime,Ertapenem,Fosfomycin, Gentamicin, Piperacillin,Colistin,Polymyxin B,Rifampin, Tetracycline,Ticarcillin, Tobramycin,Trimethoprim,Tigecycline and Meropenem according to CLSI standard.

\section{PCR}

After culturing the identified isolates of Pseudomonas aeruginosa on MacConkey agar culture medium, the genomes of all isolates were extracted using a DNA extraction kit (Bioneer Co. Korea). The polymerase chain reaction was performed with a final volume of $25 \mu \mathrm{L}$. This reaction involves $12.5 \mu$ Lof mastermix (Amplicon, Denmark), $1.5 \mu \mathrm{L}$ of forward primer, and $1.5 \mu \mathrm{Lof}$ reverse primer (offered by Bioneer Co. Korea), $2.5 \mu$ Lof DNA template, and $7 \mu \mathrm{l}$ of distilled water. The information related to primer sequence and conditions used for replication of $g y r B$, $r h l R$, exoT, lasR, PelA, and toxA genes are presented in Table 1 (3-5). The electrophoresis of PCR products was performed by $1 \%$ agarose gel, and the bands were then photographed by the Gel Doc imager. Moreover, a 50 bp marker of GenedireX Co., a joint production of Taiwan and the United States, was employed to detect PCR products.

\section{Polymerase chain reaction for identifi- cation of mexA and mexB}

After culturing the identified isolates of Pseudomonas aeruginosa on MacConkey agar culture medium, the DNAs of all isolates were extracted using a DNA extraction kit (Bioneer Co. Korea). The polymerase chain reaction was performed with a final volume of $25 \mu \mathrm{L}$. This reaction included $12.5 \mu$ Lof Master mix (Amplicon, Denmark), $1.5 \mu \mathrm{L}$ of forward primer, $1.5 \mu \mathrm{L}$ of reverse primer (offered

Table 1. Frequency of $g y r A$, rhR, exoT, lasT, Pela and toxA resistance genes in different antibiotics.

\begin{tabular}{cccccccccccccccccc}
\hline MEM & TGC & TS & TN & TC & T & RP & PRL & GM & FOT & ETP & CAZ & CPM & ATM & AK & IMI & CIP & Gene \\
\hline 0 & 32 & 32 & 4 & 10 & 32 & 32 & 4 & 5 & 18 & 21 & 4 & 28 & 8 & 2 & 2 & 3 & exo.T \\
$(0 \%)$ & $(80 \%)$ & $(80 \%)$ & $(10 \%)$ & $(25 \%)$ & $(80 \%)$ & $(80 \%)$ & $(10 \%)$ & $(12.5 \%)$ & $(45 \%)$ & $(52.5 \%)$ & $(10 \%)$ & $(70 \%)$ & $(20 \%)$ & $(5 \%)$ & $(5 \%)$ & $(7.5 \%)$ & \\
\hline 1 & 40 & 40 & 5 & 10 & 40 & 40 & 5 & 6 & 21 & 25 & 4 & 34 & 9 & 3 & 3 & 3 & rhl.R \\
$(2.5 \%)$ & $(100 \%)$ & $(100 \%)$ & $(12.5 \%)$ & $(25 \%)$ & $(100 \%)$ & $(100 \%)$ & $(12.5 \%)$ & $(15 \%)$ & $(52.5 \%)$ & $(62.5 \%)$ & $(10 \%)$ & $(85 \%)$ & $(22.5 \%)$ & $(7.5 \%)$ & $(7.5 \%)$ & $(7.5 \%)$ & \\
\hline & 40 & 40 & 5 & 10 & 40 & 40 & 5 & 6 & 21 & 25 & 4 & 6 & 9 & 3 & 1 & 3 & lasR \\
$(2.5 \%)$ & $(100 \%)$ & $(100 \%)$ & $(12.5 \%)$ & $(25 \%)$ & $(100 \%)$ & $(100 \%)$ & $(12.5 \%)$ & $(15 \%)$ & $(52.5 \%$ & $(62.5 \%)$ & $(10 \%)$ & $(15 \%)$ & $(22.5 \%)$ & $(7.5 \%)$ & $(2.5 \%)$ & $(7.5 \%)$ & \\
\hline 1 & 40 & 40 & 5 & 10 & 40 & 40 & 5 & 6 & 21 & 25 & 4 & 6 & 9 & 3 & 3 & 3 & Tox.A \\
$(2.5 \%)$ & $(100 \%)$ & $(100 \%)$ & $(12.5 \%)$ & $(25 \%)$ & $(100 \%)$ & $(100 \%)$ & $(12.5 \%)$ & $(15 \%)$ & $(52.5 \%$ & $(62.5 \%)$ & $(10 \%)$ & $(15 \%)$ & $(22.5 \%)$ & $(7.5 \%)$ & $(7.5 \%)$ & $(7.5 \%)$ & \\
\hline 0 & 37 & 37 & 4 & 10 & 37 & 37 & 4 & 5 & 20 & 23 & 4 & 5 & 8 & 2 & 2 & 3 & pel.A \\
$(0 \%)$ & $(92.5 \%)$ & $(92.5 \%)$ & $(10 \%)$ & $(25 \%)$ & $(92.5 \%)$ & $(92.5 \%)$ & $(10 \%)$ & $(12.5 \%)$ & $(50 \%)$ & $(57.5 \%)$ & $(10 \%)$ & $(12.5 \%)$ & $(20 \%)$ & $(5 \%)$ & $(5 \%)$ & $(7.5 \%)$ & \\
\hline 1 & 40 & 40 & 5 & 10 & 40 & 40 & 5 & 6 & 18 & 25 & 4 & 6 & 9 & 3 & 3 & 3 & gyrB \\
& $(100 \%)$ & $(100 \%)$ & $(12.5 \%)$ & $(25 \%)$ & $(100 \%)$ & $(100 \%)$ & $(12.5 \%)$ & $(15 \%)$ & $(45 \%)$ & $(62.5 \%)$ & $(10 \%)$ & $(15 \%)$ & $(22.5 \%)$ & $(7.5 \%)$ & $(7.5 \%)$ & $(7.5 \%)$ & \\
\hline
\end{tabular}


by Bioneer Co. Korea), $2.5 \mu$ Lof template DNA, and $7 \mu$ of distilled water. The information related to primer sequence and conditions used to replicate the mex $A$ and mexB genes are indicated in Table 1 (3-7). Electrophoresis of PCR products by $1 \%$ agarose gel. The bands were then photographed by the Gel Doc imager. Moreover, a $1 \mathrm{~kb}$ marker from GenedireX Co., a joint production of Taiwan and the United States, was employed to detect PCR products.

\section{The characteristics of the gold nano- particles}

The gold nanoparticles with a size of 40 nanometers, purity of 99.95\%, the concentration of $100 \mathrm{ppm}$, and with an international number (CAS: 744-57-5) ordered from the Nano Sadra Company (Mashhad - Iran) were used in this study (Fig. 1).

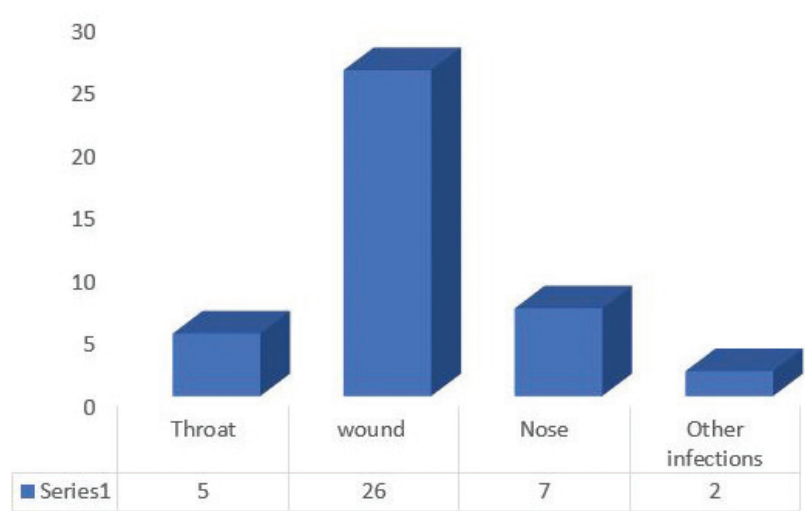

Figure 1. Frequency of clinical samples.

\section{Real-time PCR}

\section{Minimum inhibitory concentration (MIC)}

In this method, 20 out of 40 Pseudomonas aeruginosa isolates were exposed to gold nanoparticles. The broth microdilution method was used for preparing Mc Farland Standard and minimum inhibitory concentration (MIC) of growth for gold nanoparticles, and a bacterial suspension with a suitable density of Mc Farland Standard barium sulfate was employed to study the effect of antimicrobial agents. In order to prepare this standard, $5 \% \mathrm{ml}$ of $1 \%$ barium chloride was mixed with $9.95 \mathrm{ml}$ of $1 \%$ sulfuric acid. Moreover, for preparing the suspension, the bacterium was taken from a newly cultured colony and dissolved in some sterile physiological saline, and the correct density of standard turbidity(determination of microbial concentration) was identified using absorption measurements in a spectrophotometer at a wavelength of $625 \mathrm{~nm}$ between $0.8 \%$ to $1 \%$. The minimum inhibitory concentration of growth was identified using the standard method of Clinical and Laboratory Standards Institute (CLSI), and the broth dilution method was applied to determine the effect of gold nanoparticles in the case of exposure of bacteria to an antimicrobial suspension. MIC was considered as the lowest concentration of gold nanoparticles, which inhibits the growth of Pseudomonas aeruginosa isolates in culture medium was considered, and the broth microdilution method was used to assess the effect of different concentrations of gold nanoparticles on the expression of mex $A$ and mex $B$ genes of efflux pump on 20 isolates of Pseudomonas aeruginosa.

\section{RNA extraction and CDNA synthesis}

Extraction of RNA isolates treated with gold nanoparticles and non-treated in the logarithmic growth phase was performed using RNA extraction kit (Qiagen, USA) according to the instructions, and finally the DNase enzyme was employed to remove the remaining DNA, and then the concentration of RNA was determined by a nanodrop. The amount of $1 \mu \mathrm{g}$ of RNA from the sample was used for synthesizing cDNA using a QuantiTect Reverse Transcription kit (Qiagen, USA). The quantitative reverse transcription PCR (qRT-PCR) using SYBR green-contained mastermix (Applied Biosystem, UK) was applied to evaluate the mexA and mex $B$ genes of efflux pump.The materials used in $20 \mu \mathrm{L}$ of mastermix were $2 \mu \mathrm{L}$ of cDNA, $10 \mathrm{pM}$ of forward and reverse primers, and $10 \mu \mathrm{L}$ of SYBR green-contained mastermix performed on the Korean Bioneer device. The temperatures used in the $\mathrm{qPCR}$ were $90^{\circ} \mathrm{C}$ for 10 minutes, $95^{\circ} \mathrm{C}$ for 15 seconds, and $60^{\circ} \mathrm{C}$ for 1 minute performed at 40 cycles. Moreover, the G74 gene was regarded as an internal control. Finally, the relative expression of mexA and mex $B$ genes was calculated by the $\Delta \Delta$ C т method.

\section{Statistical analysis}

Statistical calculation of the present research was performed using SPSS software, and the Real-Time PCR data were analyzed by the one-way ANOVA analysis. $\mathrm{P}>0.05$ was considered statistically meaningful.

\section{Results}

\section{Clinical monitoring}

Most strains of Pseudomonas aeruginosa were isolated from wound samples, and the least strains were isolated from nasal samples (Fig. 2). The studied hospitals were

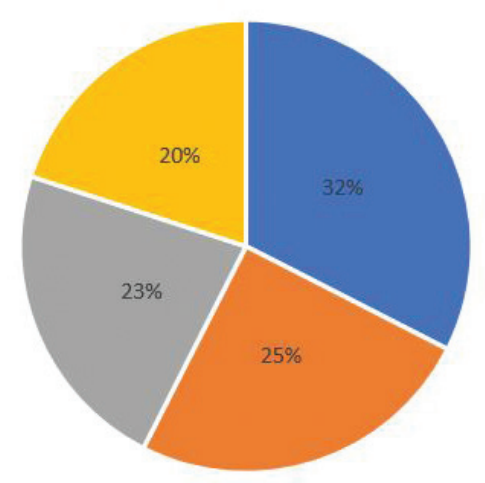

- Ostad Motahhari and Peymaniyeh = Imam Reza "- Valiasr " Omidvar

Figure 2. Distribution of clinical isolates of Pseudomonas aeruginosa in the studied hospitals. 


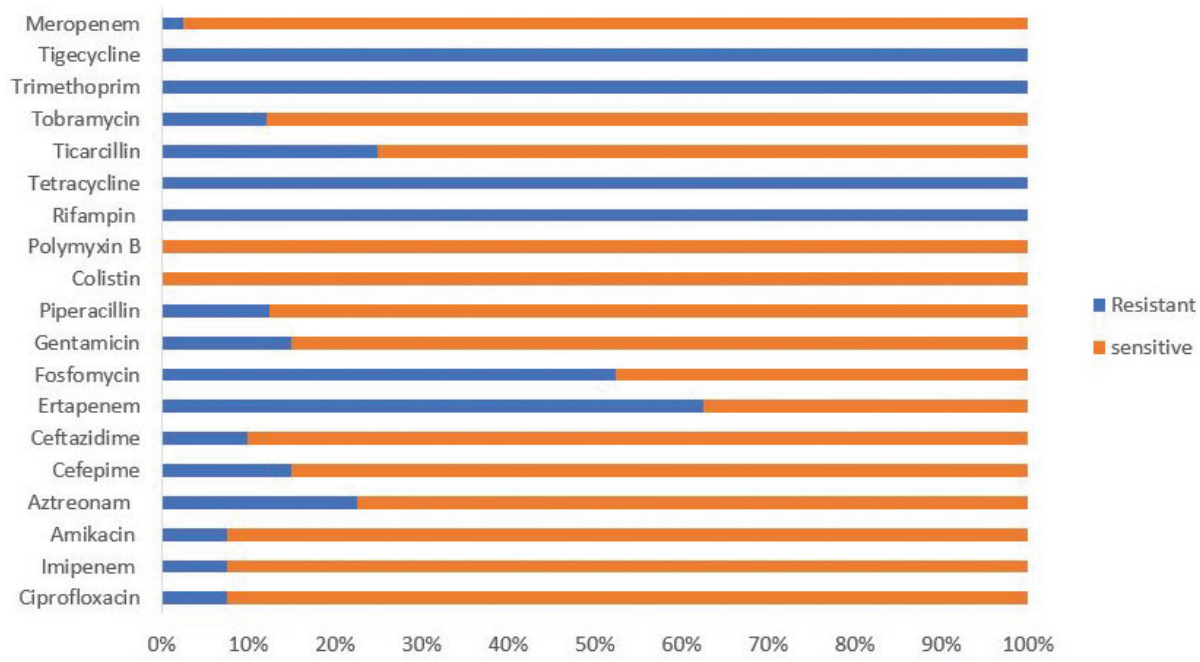

Figure 3. Susceptibility of Pseudomonas aeruginosa isolates to different antibiotics.

Ostad Motahhari and Peymaniyeh hospitals of Jahrom city in Iran,Imam Reza hospital of Lar city in Iran, Valiasr hospital of Lamerd city in Iran, and Omidvar hospital of Evaz city in Iran (Fig. 2). Forty non-duplicate strains of Pseudomonas aeruginosa were detected and identified from the studied hospitals using biochemical tests and API kits. Among 40 samples, 13 (32.5\%), 10 (25\%), 9 (22.5\%), and 8 (20\%) samples were collected from Ostad Motahhari and Peymaniyeh hospitals in Jahrom city, Imam Reza hospital in Lar city, Valiasr hospitals in Lamerd city, and Omidvar hospital in Evaz city, respectively (Fig. 3).

\section{Antibiotic susceptibility testing}

In the studied samples, the lowest levels of resistance were related to colestine $(0 \%)$, polymyxin B $(0 \%)$, meropenem (2.5\%), imipenem $(7.5 \%)$, amikacin $(7.5 \%)$, and ciprofloxacin (7.5\%), respectively. The highest resistance was observed against trimethoprim (100\%), tigecycline (100\%), tetracycline (100\%), and rifampin (100\%) (Fig. 4), respectively. According to the results, colestine and polymyxin B antibiotics are the best options for treating Pseudomonas aeruginosa infections.

\section{Results obtained from gel electropho- resis of gyrB, rhIR, exoT, lasR, Pela, and toxA genes}

The results indicated that the studied strains of Pseudomonas aeruginosa were carriers of most of these genes. The prevalence of exoT, rhlR, lasR, gyrB, pelA, and toxA genes in isolates were $32(80 \%), 40$ (100\%), 40 (100\%), 40 (100\%), 37 (92.5\%), and 40 (100\%), respectively (Figs 5-11).

Since the antibiotic resistance in Pseudomonas aeruginosa has increased today than in the past, the presence of the above genes was investigated, which are effective factors in antibiotic resistance. According to the results, the prevalence of these genes was high in clinical isolates of Pseudomonas aeruginosa, indicating their role in antibiotic resistance in the evaluated isolates (Table 1).

\section{PCR test results for $\operatorname{mex} A$ and $\operatorname{mex} B$ genes}

The presence of MexA and MexB genes in 40 Pseudomonas aeruginosa isolates was evaluated before investigating the gene expression; the results demonstrated that the pump

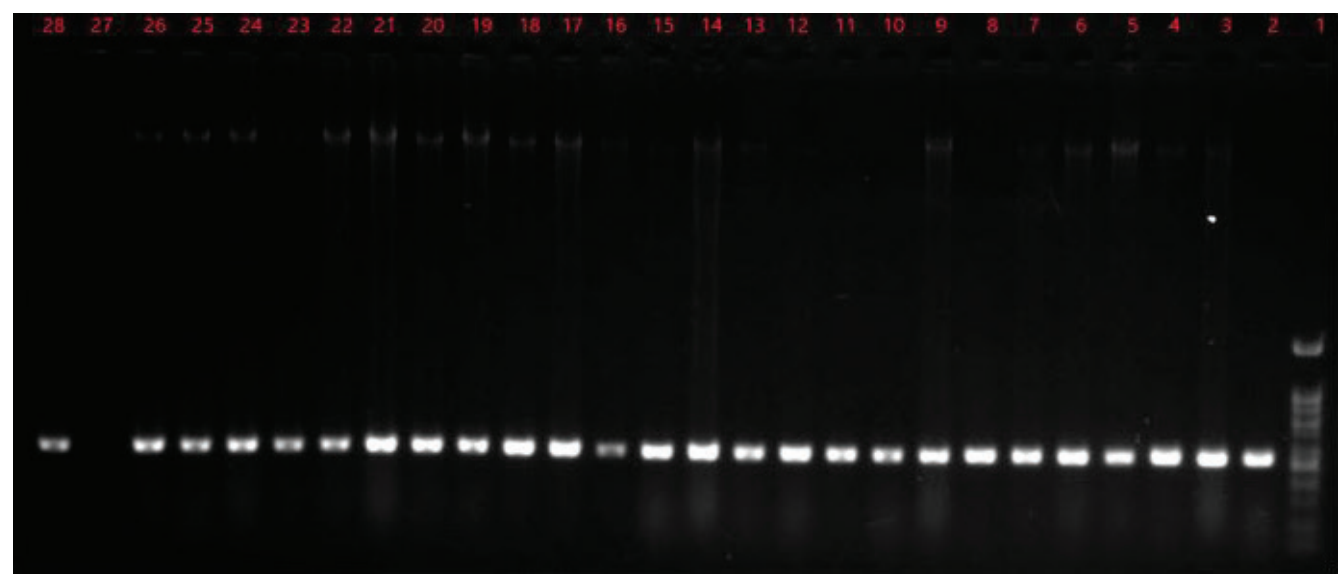

Figure 4. Results of gyrB gene replication in clinical isolates of Pseudomonas aeruginosa. Well 1: marker size 50 bp; Well 2-26: positive samples of size 222 bp; Well 27: negative control; Well 28: positive control of Pseudomonas aeruginosa PTCC 17589. 


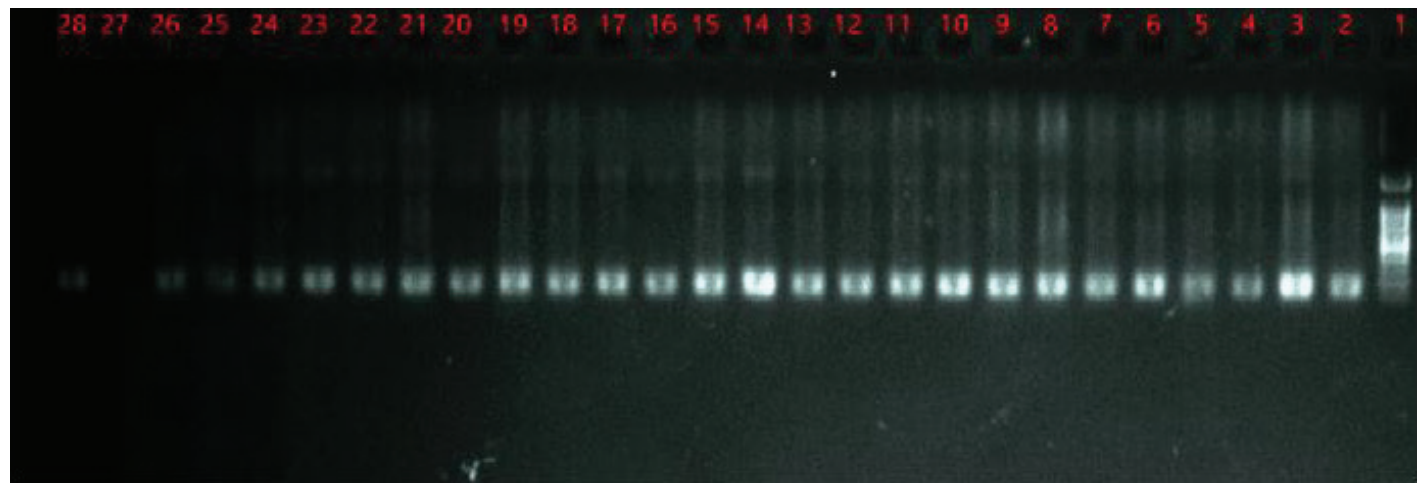

Figure 5. Results of rhlR gene replication in clinical isolates of Pseudomonas aeruginosa. Well 1: marker size 50 bp; Well 2-26: positive samples of size $133 \mathrm{bp}$; Well 27: negative control; Well 28: positive control of Pseudomonas aeruginosa PTCC 17589.

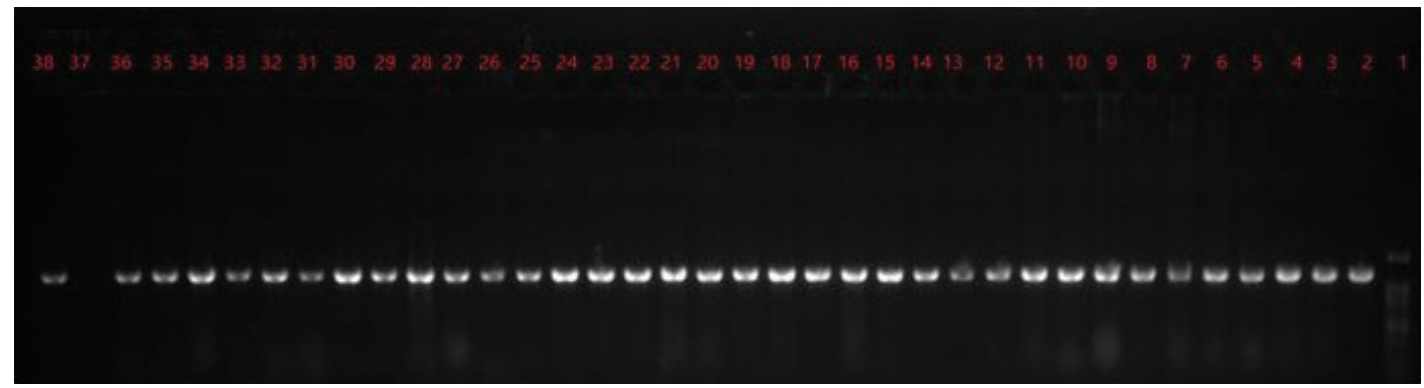

Figure 6. Results of exoT gene replication in clinical isolates of Pseudomonas aeruginosa. Well 1: marker size 50 bp; Well 2-36: positive samples of size 471 bp; Well 37: negative control; Well 38: positive control of Pseudomonas aeruginosa PTCC 17589.

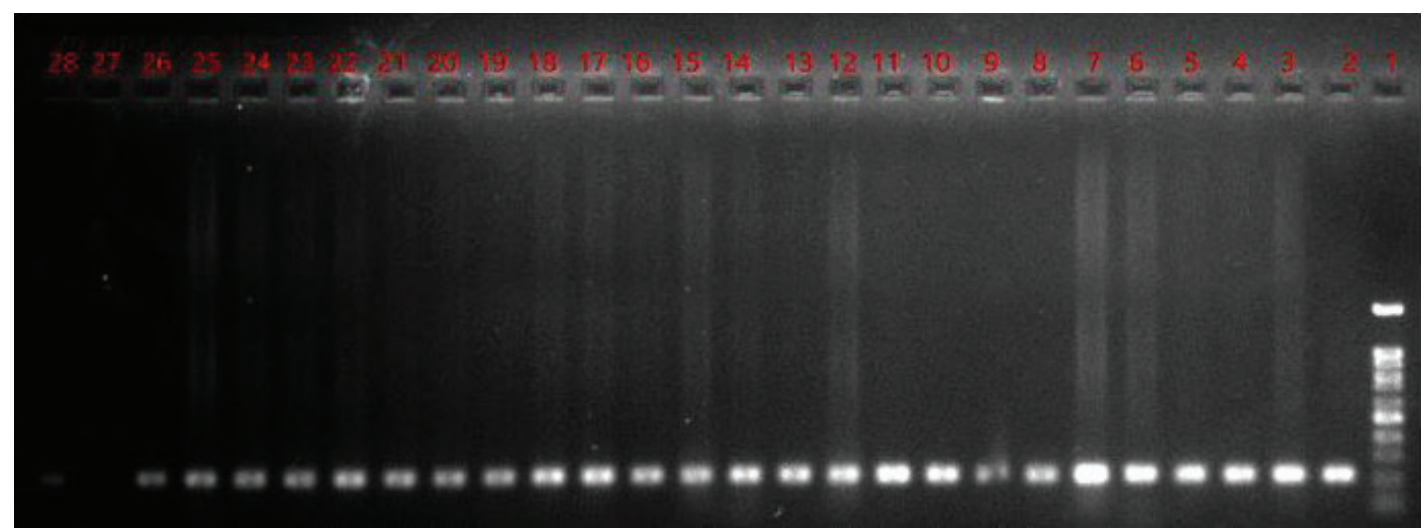

Figure 7. Results of lasR gene replication in clinical isolates of Pseudomonas aeruginosa. Well 1: marker size 50 bp; Well 2-26: positive samples of size $130 \mathrm{bp}$; Well 27: negative control; Well 28: positive control of Pseudomonas aeruginosa PTCC 17589.

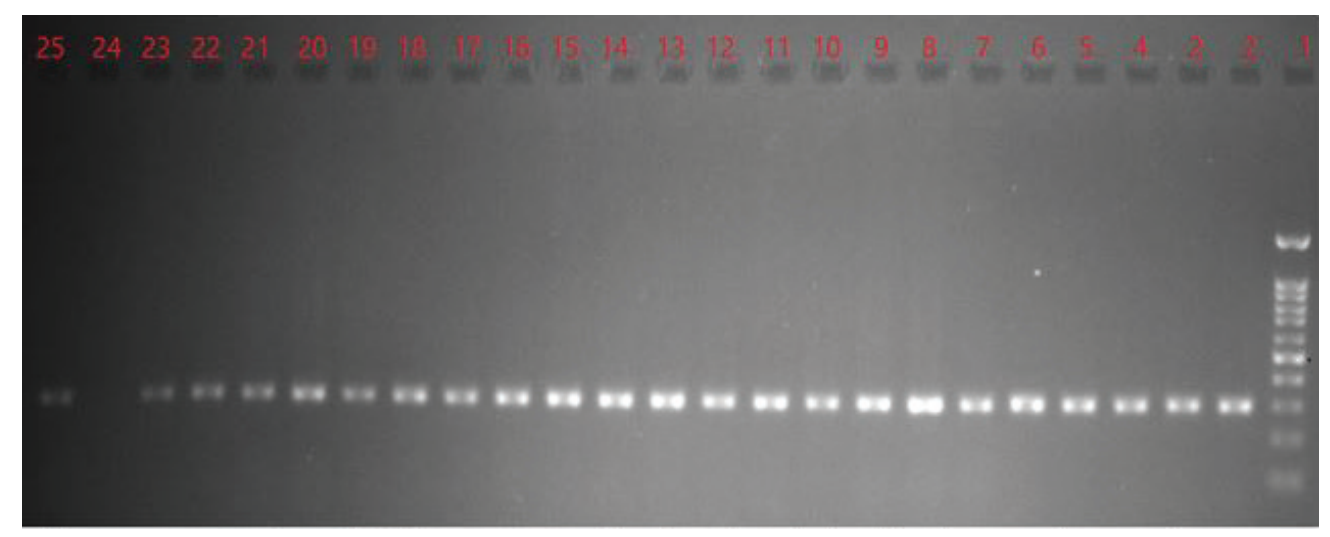

Figure 8. Results of Pela gene replication in clinical isolates of Pseudomonas aeruginosa. Well 1: marker size 50 bp; Well 2-23: positive samples of size 148 bp; Well 24: negative control; Well 25: positive control of Pseudomonas aeruginosa PTCC 17589. 


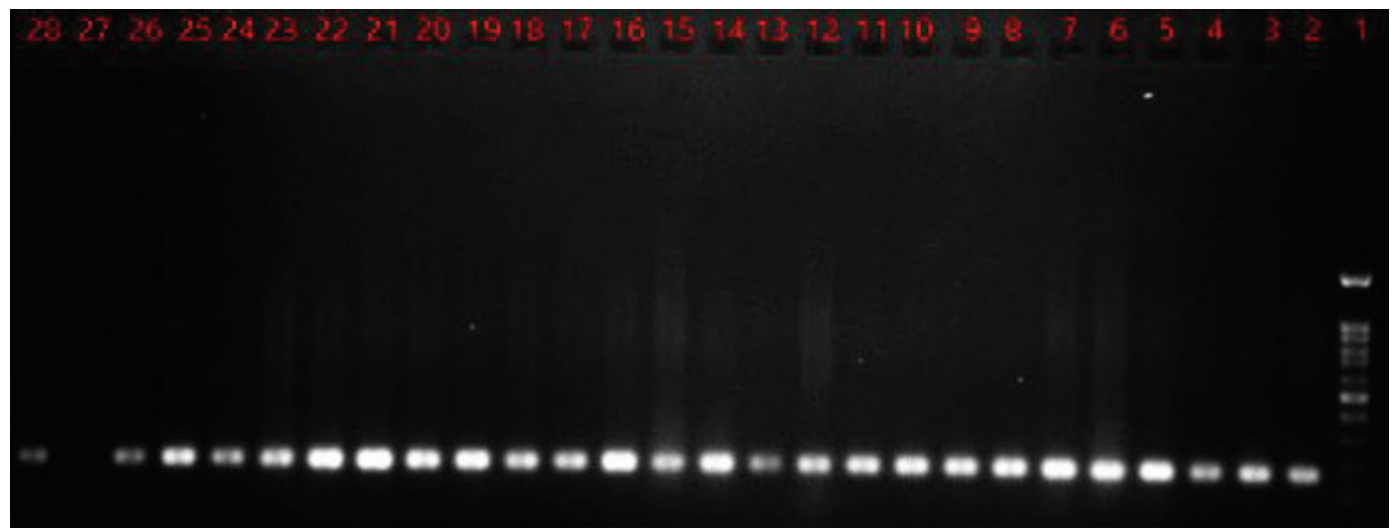

Figure 9. Results of toxA gene replication in clinical isolates of Pseudomonas aeruginosa. Well 1: marker size 50 bp; Well 2-23: positive samples of size 85 bp; Well 24: negative control; Well 25: positive control of Pseudomonas aeruginosa PTCC 17589.

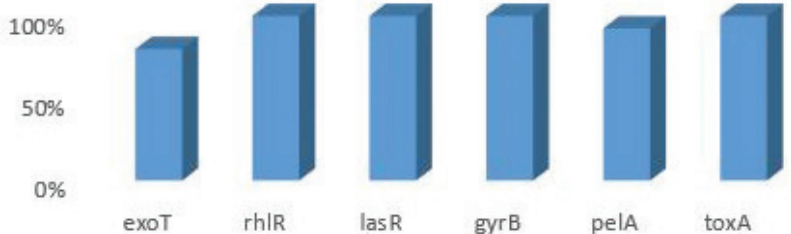

Figure 10. Percentage of Pseudomonas aeruginosa isolates carrying gyrB, rhlR, exoT, lasR, Pela, and toxA.

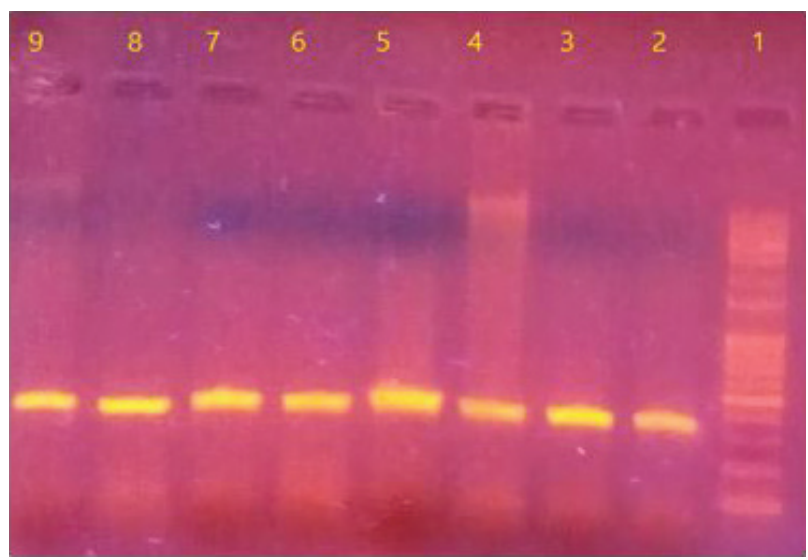

Figure 11. Results of MexA gene replication in clinical isolates of Pseudomonas aeruginosa. Well 1: marker size $1 \mathrm{~kb}$; Well 2-8: positive samples of size $316 \mathrm{bp}$; Well 9: positive control of Pseudomonas aeruginosa PTCC 17589.

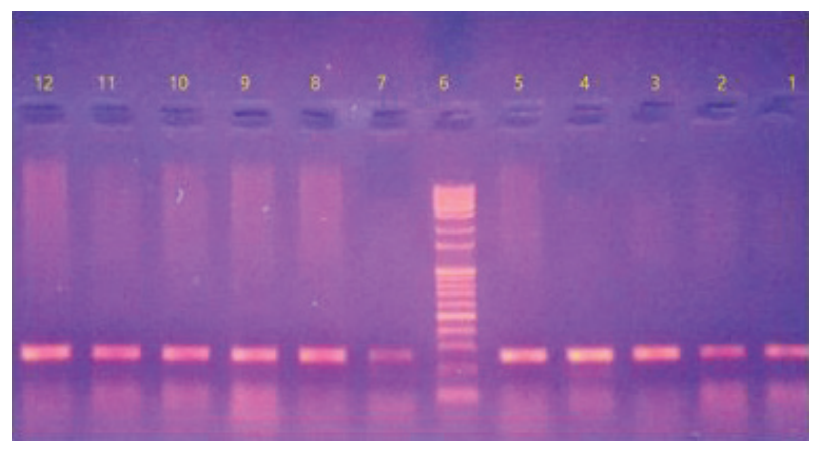

Figure 12. Results of $M e x B$ gene replication in clinical isolates of Pseudomonas aeruginosa. Well 6: marker size $1 \mathrm{~kb}$; Well 1-5 and 8-12: positive samples of size $244 \mathrm{bp}$; Well 7: positive control of Pseudomonas aeruginosa PTCC 17589. efflux genes (MexA and MexB) were present in all studied isolates (Figs 12 and 13).

\section{Expression of mex $A$ and $\operatorname{mex} B$ genes be- fore and after contact with efflux pump inhibitors through real-time PCR}

In order to compare the effect of gold nanoparticles on the expression of MexA and MexB genes after RNA extraction and cDNA synthesis, the changes of gene expression in isolates of two cell groups of "gold nanoparticles- treated" and "non-treated" was quantitatively investigated using melting curve analysis and amplification plot (Figs 14-17).

The expression rates of MexA and MexB genes in gold nanoparticle-treated isolates were meaning fully reduced compared to non-treated isolates, shown in Figs 18 and 19 , respectively.The vertical and horizontal axes of the standard curve represent CT and quantity that indicates the number of copies of the gene per dilution. It is observed in the standard curves of MexA and MexB that the $\mathrm{CT}$ evaluation factors are indirectly related to concentration and increases as concentration is decreased.

According to the results, the inhibitory effect of gold nanoparticles on MexA gene is greater compared to MexB gene.

\section{Discussion}

Since no new antibiotics are available presently to replace the existing antibiotics for Gram-negative pathogens, and there is no extensively available vaccine against these infections, just one way to mitigate the effects of infections is to control their spread that can only be achieved in the case of fully understanding of causes, dynamics, and complexity of the prevalence of these organisms. The objective of the present studies was to contribute to this knowledge by studying Pseudomonas aeruginosa, which is the cause of nosocomial infections in burn-injured patients.

Much research has been carried out on the resistance of pseudomonas, the results of which are different in terms of time and place. In the present study, antibiotic resistance of 40 isolated strains from Omidvar hospital of Evaz city, Valiasr hospital of Lamerd city, Imam Reza hospital of Lar city, 


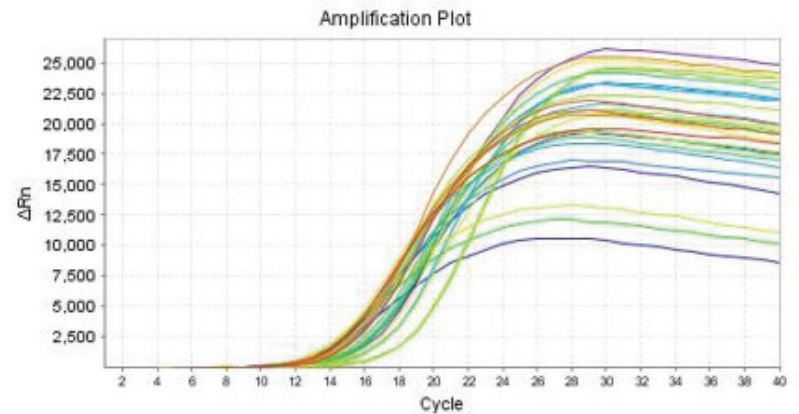

Figure 13. Results of MexA gene amplification curve in Real Time PCR by cycle.

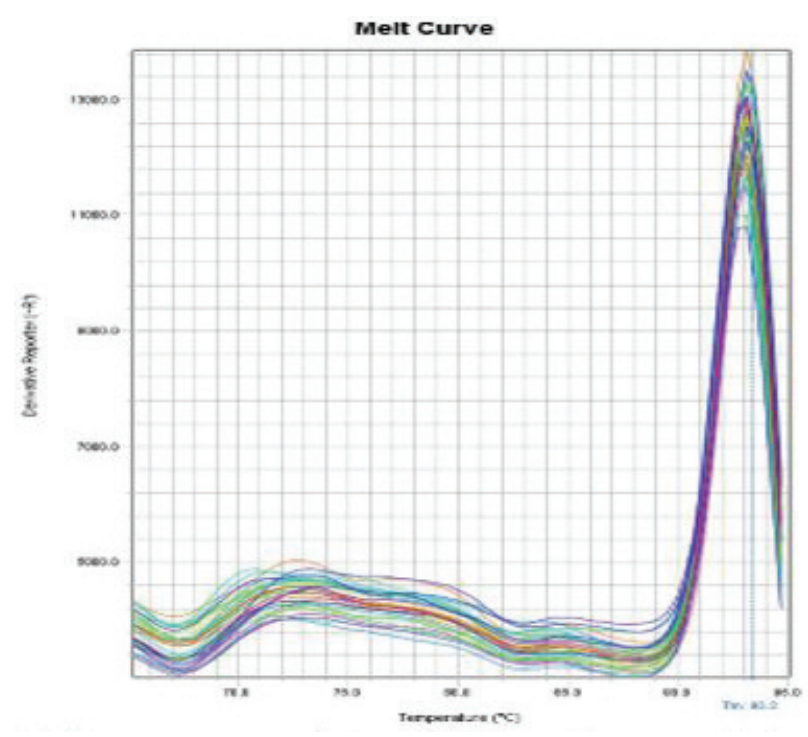

Figure 14. Melting curve analysis to ensure the specificity of the amplified fragments of the MexA gene; the measured curves of the gene in all samples are consistent and in the form of single-peak.

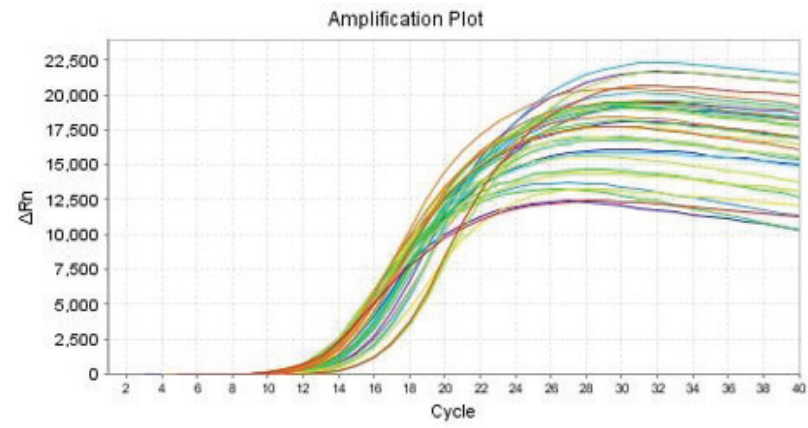

Figure 15. Melting curve analysis to ensure the specificity of the amplified fragments of the MexB gene that the measured curves of the gene in all samples are consistent and single-peaked.

and Ostad Motahhari and Peymaniyeh hospitals of Jahrom city were evaluated; the resistance rates was as follows:Colestine $0 \%$, ciprofloxacin $3(7.5 \%)$, imipenem 3 (7.5\%), amikacin $3(7.5 \%)$, aztreonam $9(22.5 \%)$, cefepime $6(15 \%)$, ceftazidime $40(100 \%)$, ertapenem 25 (62.5\%), fosfomycin 21 (52.5\%), gentamicin $6(15 \%)$, piperacillin $5(12.5 \%)$, polymyxin B 0\%, Rifampin 40 (100\%), tetracycline 40 (100\%), ticarcillin 10 (25\%), tobramycin 5 (12.5\%), trimethoprim 40 (100\%), tigecycline 40 (100\%), and meropenem 1 (5/2\%).

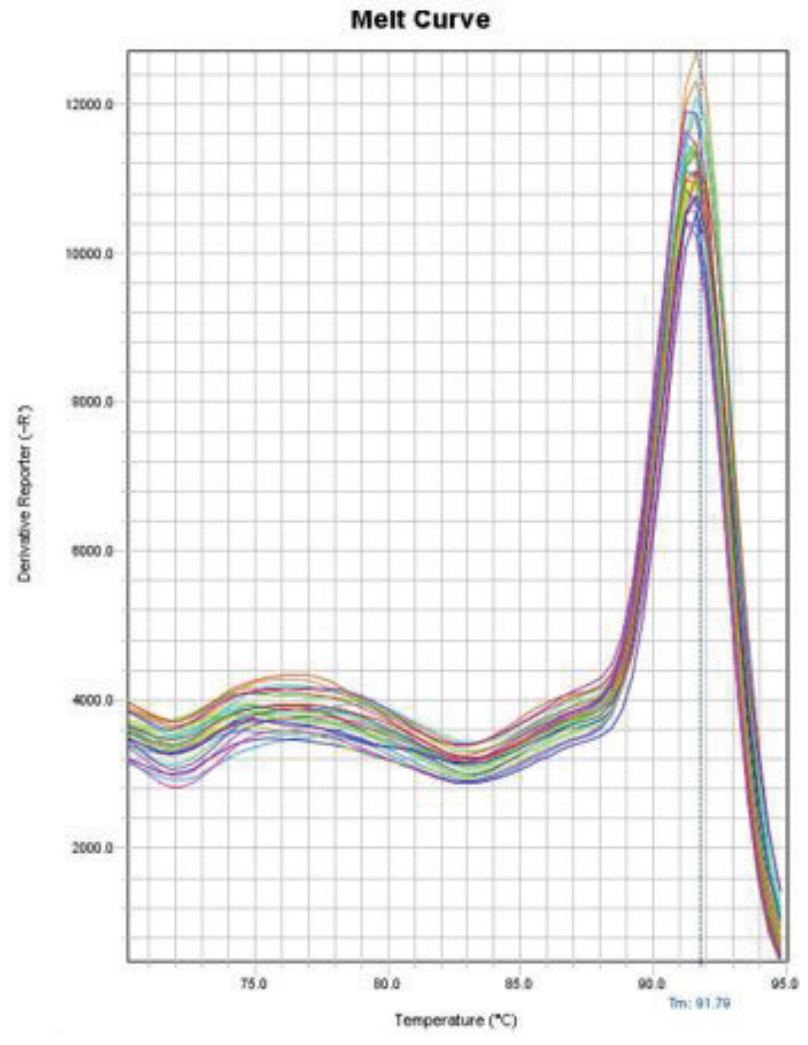

Figure 16. Melting curve analysis to ensure the specificity of the amplified fragments of the MexB gene; the measured curves of the gene in all samples are consistent and in the form of single-peak.

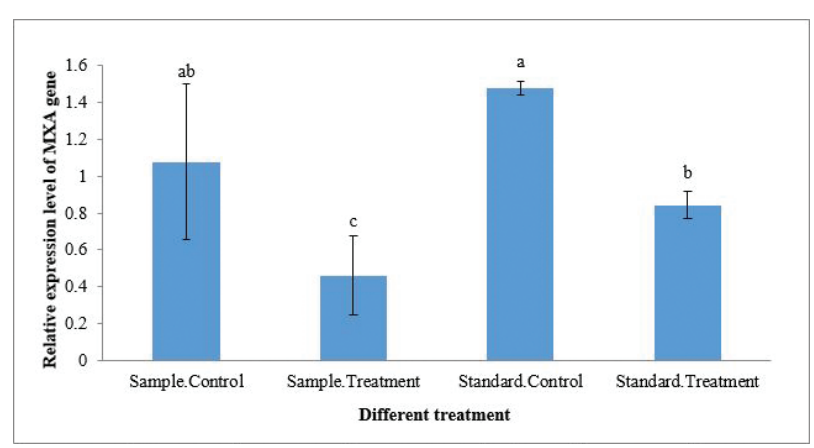

Figure 17. The expression rates of MexA gene in the gold nanoparticles-treated samples and non-treated samples; a significant decrease in gene expression was observed between treated and non-treated samples in comparison with the control group. The G74 gene was used as an internal control.

In a study conducted by Shahid et al on Pseudomonas aeruginosa strains at the burn care units, the lowest and highest resistances were against the tetracycline (0\%) and gentamicin (90\%), respectively(Shahid et al. 2003). According to the present study, the results indicated an increase in drug resistance for the reason of overuse over a period of time. According to the results of the present research as well as the literature, the colestine and polymyxin B are the best therapeutic options against Pseudomonas aeruginosa infections.

Pseudomonas aeruginosa is a stubborn microorganism in terms of resistance to various antibiotics and possesses three main mechanisms of limited adsorption resistance 


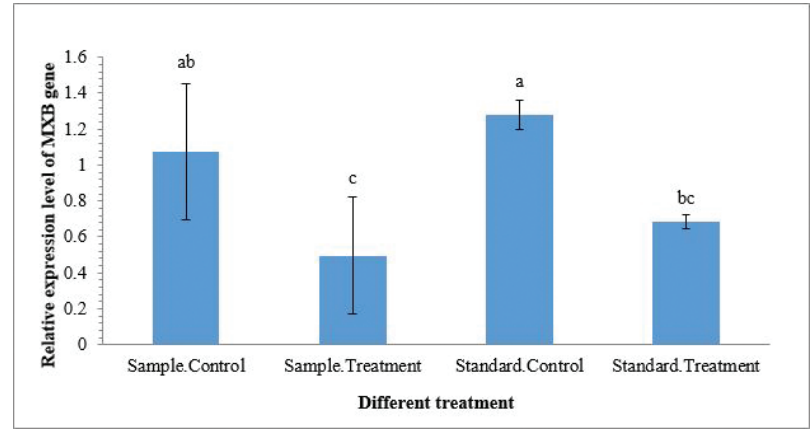

Figure 18. The expression rates of $M e x B$ gene in the gold nanoparticles-treated samples and non-treated samples; a significant decrease in gene expression was observed between treated and non-treated samples in comparison with the control group. The G74 gene was used as an internal control.

and efflux, drug inactivation, and change in targets (Brinkman et al. 2000). Efflux pumps play an important role in the resistance of Pseudomonas aeruginosa. All antibiotic classes except polymyxins are sensitive to excretion by one or more of these efflux systems.

Among these systems, the MexAB-oprM is of great importance nowadays, which is responsible for the excretion of beta-lactams, quinolones, and an extensive range of anti-microorganisms. In the present article, the presence of mexA and mexB genes was investigated. The Mex$A B$-oprM genes are chromosomal genes present in all wild strains, and their absence can be attributed to mutations. Arabestani et al. concluded that mexAB-oprM genes were present in $100 \%$ isolates of Pseudomonas aeruginosa(Arabestani et al. 2015). Moreover, Elgravi reported the presence of mexAB-oprM of septicemic Pseudomonas aeruginosa with the frequency of $100 \%$ of samples for both mex $A$, mex $B$ operon genes indicating the chromosomal nature of the mexAB-oprM efflux pump genes (Al-Grawi 2012). In the present study, the presence of MexA and $M e x B$ genes in 40 isolates of Pseudomonas aeruginosa was evaluated before investigating the gene expression; the results demonstrated that the MexA and MexB efflux pump genes were present in all studied isolates, which was in line with the results of previous investigations.

Many investigation sindicate that the antimicrobial properties of antibiotics can be increased by destroying efflux pumps. The inhibitors of these pumps can be employed to disrupt the function of drug efflux pumps. Nowadays, various inhibitors, such as alanine-arginine beta-naphthylamide,can change the minimum concentration of inhibitors by excreting toxic substances. Gold nanoparticles currently have extensive applications in biomedicine, including bioimaging, gene transfer, drug delivery, plasmonic biosensing, colorimetric assays, tissue engineering, imaging therapy, and cancer therapy. The antibacterial mechanism for gold nanoparticles depends on their size; smaller gold nanoparticles create irreversible pores as they move through the bacterial cell membrane (Zheng et al. 2017). The antibacterial activity of gold nanoparticles involves several mechanisms, such asthe pres-

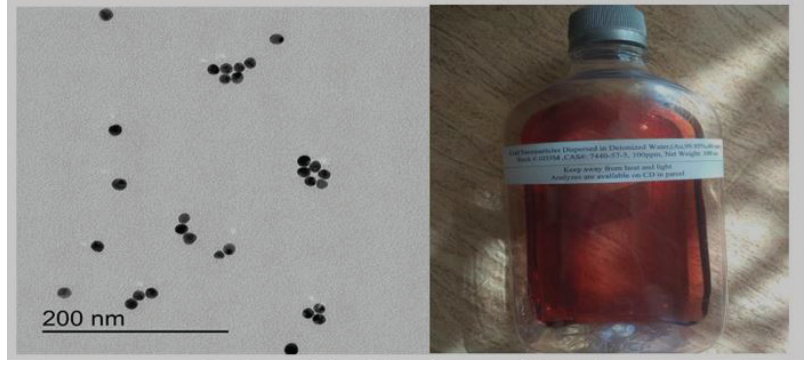

Figure 19. The characteristics of the gold nanoparticles used.

ence of a strong positive charge on gold nanoparticles that increases the tendency of these nanoparticles toward the negative charge of bacteria, which in turn disrupts the integrity of the cell membrane and leads to cell rupture. In the present study, the expression of MexA and MexB genes before and after contact with efflux pump inhibitors (gold nanoparticles) was investigated by the Real-Time PCR technique. Unfortunately, not much research has been conducted on the effect of gold nanoparticles on the function of efflux pump genes, but many studies have been carried out on the use of other nanoparticles on these genes.

Aria et alstudied the effect of vanilla and the applicaiton of gold nanoparticles on multidrug-resistant isolates of Pseudomonas aeruginosa and concluded that treatment with gold nanoparticles reduced the expression of mexA and mexB genes, which is in line with the results of the present paper(Arya et al. 2019), in which a reduction in the expression of mex $A$ and mexB genes was observed in the gold nanoparticle-treated strains.

Furthermore, Mohammadipour et al. conducted a piece of researcher garding the effect of encapsulated silybin in nanoparticles on the expression of oprM gene in resistant strains of Pseudomonas aeruginosa and concluded thata decrease in oprM expression in resistant isolates caused a reduction in mexAB-oprM, mexXY-oprM on the cell surface and an increase in the sensitivity to antibiotics that were in line with the results of the present research (Mohammadipour et al. 2017). The gold nanoparticles reduce the expression of mexA and mexB genes and increase antibiotic sensitivity, indicating the positive effect of gold nanoparticles on the antibiotic-resistant isolates of Pseudomonas aeruginosa.

Rudbaraki conducted an study on the change of mexB gene expression in ciprofloxacin-resistant Pseudomonas aeruginosa isolates under treatment with encapsulated silibinin in nanoparticles and concluded that silibinin increases the capability of ciprofloxacin in inhibiting the growth of Pseudomonas aeruginosa by reducing the expression of genes involved in efflux pump systems, such as mexB gene (Ahmadi et al. 2017).

In the present study, the expression rate of MexA gene in gold nanoparticle-treated isolates was significantly reduced thanin non-treated isolates. An investigation on $M e x B$ expression indicated that gold nanoparticle-treated samples showed a significant decrease in expression compared to non-treated samples. According to the findings, the inhibitory effect of gold nanoparticles on MexA gene is higher than this effect on MexB gene. 


\section{Conclusion}

The MexAB-oprM system is one of the main factors in the resistance of Pseudomonas aeruginosa. The results of the present investigation indicated that gold nanoparticles re-

\section{References}

Ahmadi Roudbaraki Z, Ranji N, Soltani Tehrani B (2007) Deregulation of mexB Gene in Ciprofloxacin Resistant Isolates of Pseudomonas aeruginosa Treated with Silibinin-Encapsulated in Nanoparticles. JBUMS 19(11): 42-49.

Al-Grawi IGA (2012) Occurrence of MexAB-OprM ef flux pump operon on septicemic Pseudomonas aeruginosa chromosome. Iraqi Academic Scientific Journal 11(1): 97-102.

Arabestani MR, Rajabpour M, YousefiMashouf R, Alikhani MY, Mousavi SM (2015) Expression of efflux pump MexAB-OprM and OprD of Pseudomonas aeruginosa strains isolated from clinical samples u sing qRT-PCR. Archives of Iranian medicine 18(2) 102-108.

Arya SS, Sharma MM, Das RK, Rookes J, Cahill D, Lenka SK (2019) Vanillin mediated green synthesis and application of gold nanoparticles for reversal of antimicrobial resistance in Pseudomonas aeruginosa clinical isolates. Heliyon 5(7): 1-11. https://doi.org/10.1016/j.heliyon.2019.e02021

Brinkman FS, Bains M, Hancock RE (2000) The amino terminus of Pseudomonas aeruginosa outer membrane protein OprF forms channels in lipid bilayer membranes: correlation with a three dimensional model. Journal of Bacteriology 182: 5251-5255. https://doi. org/10.1128/JB.182.18.5251-5255.2000

Gellatly SL, Hancock RE (2013) Pseudomonas aeruginosa: new insight s into pathogenesis and host defenses. Pathogens and Disease 67(3) 159-173. https://doi.org/10.1111/2049-632X.12033

Ghazi M, Isadyar M, Gachkar L, Mahmoudi S, Goudarzi H, Eslami G, Pourakbari B, Fallah F (2012) Serum levels of mannose-binding lectin and the risk of infection in pediatric oncology patients with chemotherapy. Journal of Pediatric Hematology/Oncology 34(2): 128-130. https://doi.org/10.1097/MPH.0b013e31822bf7d3

Goli HR, Nahaei MR, Rezaee MA, Hasani A, Kafil HS, Aghazadeh M, Nikbakht M, Khalili Y (2018) Role of MexAB-OprM and MexXY OprM efflux pumps and class 1 integrons in resistance to antibiotics i $\mathrm{n}$ burn and Intensive Care Unit isolates of Pseudomonas aeruginosa. Journal of Infection and Public Health 1, 1(3): 364-372. https://doi. org/10.1016/j.jiph.2017.09.016 duce the number of active efflux pumps on the cell surface by reducing the expression of mexA and mexB genes so that a decrease in the level of these efflux pumps reduces the excretion of antibiotics from the cell and subsequently causes lower concentrations of antibiotics to be able to kill cells.

Jafari M, Fallah F, Borhan RS, Navidinia M, Karimi A, Tabatabaei SR (2013) The first report of CMY, aac (6')-Ib and 16S rRNA methylase genes among Pseudomonas aeruginosa Isolates from Iran. Archives Of Pediatric Infectious Disease 1(3): 109-112. https://doi. org/10.5812/pedinfect.11392

Kalishwaralal K, Deepak V, Ram Kumar Pandian S, Kottaisamy M, BarathmaniKanth S, Kartikeyan B, Gurunathan S (2010). Biosynthesis of silver and gold nanoparticles using Brevibacterium casei. Colloids Surf B Biointerfaces 1, 77(2): 257-262. https://doi.org/10.1016/j. colsurfb.2010.02.007

Mohammadipour A, Ranji N, Asadpour L (2017) The Effect of Silybin Encapsulated in Nanoparticles on oprM Gene Expression in Drug Resistant Isolates of Pseudomonas aeruginosa. Journal of Arak University Medical Sciences. 20(5): 79-88.

Munita JM, Arias CA (2016) Mechanisms of antibiotic resistance. Microbiology Spectrum 4(2): 10. https://doi.org/10.1128/microbiolspec.VMBF-0016-2015

Nehme D, Poole K (2007) Assembly of the MexAB-OprM multidrug pump of Pseudomonas aeruginosa: component interactions defined by the study of pump mutant suppressors. Journal of Bacteriology 189(17): 6118-6127. https://doi.org/10.1128/JB.00718-07

Rai M, Yadav A, Gade A (2009) Silver nanoparticles as a new generation of antimicrobials. Biotechnology Advances 27(1): 76-83. https://doi. org/10.1016/j.biotechadv.2008.09.002

Sadikot RT, Blackwell TS, Christman JW, Prince AS (2005) Pathogen-host interactions in Pseudomonas aeruginosa pneumonia. American Journal of Respiratory and Critical Care Medicine 171(11): 1209-1223. https://doi.org/10.1164/rccm.200408-1044SO

Shahid M, Malik A, Sheeba (2003) Multidrug-resistant Pseudomonas aeruginosa strains harbouring R-plasmids and $A m p C \beta$-lactamases isolated from hospitalised burn patients in a tertiary care hospital of North India. FEMS Microbiology Letters 228(2): 181-186. https:// doi.org/10.1016/S0378-1097(03)00756-0

Zheng K, Setyawati MI, Leong DT, Xie J (2017) Antimicrobial Gold Nanoclusters. ACS Nano 11(7): 6904-6910. https://doi.org/10.1021/ acsnano.7b02035 